\title{
Computed tomography assessment of peripheral traction bronchiolectasis: impact of minimal intensity projection
}

\author{
To the Editor:
}

The Fleischner Society white paper and the American Thoracic Society (ATS)/European Respiratory Society (ERS)/Japanese Respiratory Society (JRS)/Latin American Thoracic Association (ALAT) guidelines recently redefined the computed tomography (CT) scanning patterns of usual interstitial pneumonia (UIP) $[1,2]$. Both publications confirmed honeycombing as the cornerstone of UIP pattern diagnosis, and introduced peripheral traction bronchiolectasis (PTB) as a key feature of the new "probable UIP" category. Therefore, improving the distinction between these two features may be critical, especially when the clinical likelihood of idiopathic pulmonary fibrosis (IPF) is uncertain; a lung biopsy should be discussed for patients with PTB without honeycombing, but is not recommended for patients presenting with clear honeycombing and typical UIP pattern [2].

The Fleischner Society white paper and the ATS/ERS/JRS/ALAT guidelines both stress the importance of the CT technical parameters for the image acquisition, but only the first publication provides indications for the reading step. It proposes the use of cine mode and multiplanar (MPR) reformations, as well as post-processing with minimum intensity projection (MinIP) to help differentiate honeycombing from peripheral bronchiectasis [1].

Thus, we evaluated whether the use of MinIP improves inter-reader agreement for the identification of PTB and honeycombing. As a secondary objective, we tested whether the use of MinIP improves inter-reader agreement for the assessment of CT scanning patterns of UIP, according to the 2018 ATS/ ERS/JRS/ALAT guidelines [2].

This prospective study was conducted in four reference hospitals for interstitial lung disease in the Paris area, France. Approval was obtained from the institutional ethics committee (CPP Ile-de-France-X, November 2011); all included patients signed informed consent.

All patients presenting with suspected idiopathic interstitial pneumonia whose cases were discussed for the first time during local multidisciplinary discussions (MDD) from January 2014 to December 2015 were screened for the study. Patients were included if no specific pulmonary diagnosis had been made before the MDD and if a recent ( $\leqslant 3$ months) chest CT with contiguous thin sections $(\leqslant 1.25 \mathrm{~mm})$ showing fibrotic features (architectural distortion, traction bronchiectasis/bronchiolectasis, honeycombing) was available. 168 patients were eligible and all agreed to participate in the study. 1 year after the initial MDD, 16 patients were lost to follow-up; moreover, CT images were missing for 11 patients. These 27 patients were excluded and 141 patients were finally included. The clinical diagnosis established by the second MDD after 1 year was recorded.

Four thoracic radiologists (including two specialists in interstitial lung diseases) and two pulmonologists (specialists in interstitial lung diseases) reviewed the chest CTs. Honeycombing was defined according to the Fleischner Society glossary [3], and PTB as peripheral bronchiectasis abutting the pleura. The readers participated in a training session before starting their readings. Readers reviewed each case twice on a picture archiving and communication system workstation, using cine mode and MPR only (method 1), and then using additional 3-5 mm MinIP post-processing (method 2) during a second session, after a minimal 2-week interval to avoid recall bias. For each session, readers were asked to assess 1) the presence/

@ERSpublications

On chest CT, post-processing with minimal intensity projection does not improve inter-reader agreement for the identification of peripheral traction bronchiolectasis and honeycombing in patients with suspected idiopathic interstitial pneumonia. http://bit.ly/35p8NlQ

Cite this article as: de Margerie-Mellon C, Belin L, Boussouar S, et al. Computed tomography assessment of peripheral traction bronchiolectasis: impact of minimal intensity projection. Eur Respir J 2020; 55: 1901388 [https://doi.org/10.1183/13993003.01388-2019]. 
absence of PTB and honeycombing in three areas (table 1) and 2) the CT pattern of UIP according to the ATS/ERS/JRS/ALAT guidelines [2].

For the inter-reader analysis, we calculated Fleiss's $\kappa$ coefficient [4] to estimate agreement between more than two readers and its bootstrapped confidence interval. Agreements were calculated per area and per patient, considering that a patient presented the trait "presence of honeycombing" or "presence of PTB" when at least one area presented the trait. To compare $\kappa$ coefficients, we calculated the bootstrapped confidence interval of the difference of $\kappa$ between groups and the corresponding $p$-value. We used the following R packages: the "rel" package (version 1.3-1) and the "boot" package (version 1.3-18). A p-value $<0.05$ was taken to represent statistical significance. Statistical analysis was performed with $\mathrm{R}$ version 3.3.2.

Among the 141 included patients, 109 (77\%) were male. Median age (range) was 68 (24-91) years. After a 1-year follow-up, 28 (20\%) patients had undergone a lung biopsy. The 1-year clinical diagnoses were IPF in $66(47 \%)$ patients, including $12(9 \%)$ patients who had undergone a lung biopsy; nonspecific interstitial pneumonia (NSIP) in 19 (13\%) patients; and other specific pneumonia in four (3\%) patients. Finally, 52 patients $(37 \%)$ remained unclassified.

$\kappa$ coefficients $(95 \%$ CI) for inter-reader agreement over the six readers for the identification of PTB, for the identification honeycombing and for the CT patterns of UIP with methods 1 and 2 are presented in table 1. Inter-reader agreement ranged from 0.44 to 0.65 , and was not significantly different between method 1 and method 2 for all three parameters ( $\mathrm{p}=0.397-0.945)$.

With both methods, readers identified PTB in $76-100 \%$ of IPF patients diagnosed on a lung biopsy, and in $73-96 \%$ of other IPF patients. Similarly, honeycombing was identified in $17-50 \%$ of patients when IPF diagnosis relied on a lung biopsy, and in 59-91\% of patients when the IPF diagnosis was made without histological evidence. Regarding patients with NSIP, PTB was identified in $37-84 \%$ of patients and honeycombing in $16-47 \%$.

PTB is an early hallmark of pulmonary fibrosis; however, honeycombing and PTB commonly coexist and can be confused. Post-processing with MinIP allows detection of low-density structures in a given volume, by projecting the voxel with the lowest attenuation value on every view throughout the volume onto a two-dimensional image. Theoretically, it is an optimal tool to visualise the subtle differences between endobronchial air and lung parenchyma [5]. Therefore, we hypothesised that inter-reader agreement for the identification of PTB and honeycombing would be increased using MiniP post-processing, by improving the distinction between the two CT features.

However, based on 141 CT examinations and six readers, our study was unable to demonstrate the superiority of MinIP post-processing over conventional reading methods to identify these signs reliably. Inter-reader agreement for PTB was only moderate, and tended to be lower than the inter-reader agreement for honeycombing, which was moderate to substantial, whatever the reading method.

\begin{tabular}{|c|c|c|c|}
\hline & Method $1^{\#}$ & Method 2ף & p-value \\
\hline \multicolumn{4}{|c|}{ Peripheral traction bronchiolectasis } \\
\hline Superior zone & $0.52(0.43-0.59)$ & $0.53(0.44-0.61)$ & 0.840 \\
\hline Middle zone & $0.54(0.46-0.61)$ & $0.50(0.43-0.57)$ & 0.684 \\
\hline Inferior zone & $0.44(0.36-0.53)$ & $0.45(0.36-0.54)$ & 0.945 \\
\hline Per patient & $0.49(0.39-0.58)$ & $0.48(0.38-0.57)$ & 0.830 \\
\hline \multicolumn{4}{|l|}{ Honeycombing } \\
\hline Superior zone & $0.62(0.53-0.70)$ & $0.64(0.55-0.72)$ & 0.812 \\
\hline Middle zone & $0.65(0.57-0.72)$ & $0.61(0.52-0.69)$ & 0.462 \\
\hline Inferior zone & $0.63(0.55-0.70)$ & $0.60(0.51-0.67)$ & 0.539 \\
\hline Per patient & $0.60(0.53-0.68)$ & $0.57(0.49-0.64)$ & 0.455 \\
\hline \multicolumn{4}{|c|}{ CT patterns of UIP ${ }^{+}$} \\
\hline Per patient & $0.55(0.49-0.60)$ & $0.52(0.46-0.57)$ & 0.397 \\
\hline
\end{tabular}

Superior zone: above carina; inferior zone: under inferior pulmonary veins; middle zone: in between superior and inferior zones; CT: computed tomography; UIP: usual interstitial pneumonia. \#: reading using cine mode and multiplanar reformations only; ${ }^{\text {? }}$ : reading using cine mode, multiplanar reformations and minimal intensity projection post-processing: ${ }^{+}$: CT patterns of UIP (UIP, probable UIP, indeterminate for UIP, alternative diagnosis). 
These results may be explained by the difficulty in differentiating PTB from honeycombing on its peripheral location. Indeed, these two features seem closely intertwined. It has been shown that honeycombing on CT correspond, at least partly, to traction bronchiolectasis on pathology [6-8]. In line with PICIUCCHI et al. [9], we think that PTB and honeycombing are different faces of the same continuous spectrum of lung remodelling, and therefore, they are intrinsically indistinguishable in many cases.

PTB and honeycombing are key components of the CT patterns of UIP according to the 2018 guidelines [2]. As a consequence, it was expected that the inter-reader agreement for the CT patterns of UIP would also be moderate, and not improved by the use of MiniP reformations. The recent guidelines were developed to overcome some limitations of the previous recommendations, and the category "probable UIP" was created. PTB is one of its important features. However, PTB was also identified in up to $84 \%$ of our NSIP cases. This finding highlights the importance of analysing CT features in the light of the clinical context, especially age and sex, during MDDs.

To conclude, there was no improvement of the inter-reader agreement with MinIP for the identification of PTB and honeycombing in our study, nor was there improvement of the inter-reader agreement for the assessment of CT patterns of UIP. We believe that radiologists should choose whether to use MiniP reformations for the assessment of fibrotic lung diseases according to their habits and personal preferences.

Constance de Margerie-Mellon $\oplus^{1}$, Lisa Belin ${ }^{2}$, Samia Boussouar ${ }^{3}$, Philippe Khafagy ${ }^{4}$, Marie-Pierre Debray ${ }^{5}$, Kim Levand ${ }^{6}$, Marie-Laure Chabi ${ }^{7}$, Antoine Khali ${ }^{5}$, Amira Benattia ${ }^{8}$, Dominique Israël-Biet ${ }^{8}$, Bruno Crestani ${ }^{9}$, Hilario Nunes ${ }^{10}$, Jacques Cadranel ${ }^{11}$, Philippe Grenier ${ }^{3}$, Dominique Valeyre ${ }^{10}$, Jean-Marc Naccache $\oplus^{11}$ and Pierre-Yves Brillet ${ }^{6}$

${ }^{1}$ Université de Paris, Assistance Publique-Hôpitaux de Paris, Hôpital Saint-Louis, Service de Radiologie, Paris, France. ${ }^{2}$ Sorbonne Université, INSERM UMR 1136, Institut Pierre Louis d'Epidémiologie et de Santé Publique, Assistance Publique-Hôpitaux de Paris, Hôpitaux Universitaires Pitié-Salpétrière-Charles Foix, Département Biostatistique, Santé Publique et Information Médicale, Paris, France. ${ }^{3}$ Sorbonne Université, Assistance Publique-Hôpitaux de Paris, Hôpitaux Universitaires Pitié-Salpétrière-Charles Foix, Service de Radiologie, Paris, France. ${ }^{4}$ Groupe Hospitalier le Raincy Montfermeil, Montfermeil, France. ${ }^{5}$ Université de Paris, INSERM UMR 1152 "Physiopathologie et épidémiologie des maladies respiratoires", Assistance Publique-Hôpitaux de Paris, Hôpital Bichat-Claude Bernard, Service de Radiologie, Paris, France. ${ }^{6}$ Université Paris 13, INSERM UMR 1272 "Hypoxie et Poumon", Assistance PubliqueHôpitaux de Paris, Hôpital Avicenne, Service de Radiologie, Bobigny, France. ${ }^{7}$ Hôpital Foch, Service de Radiologie, Suresnes, France. ${ }^{8}$ Université de Paris, Assistance Publique-Hôpitaux de Paris, Hôpital Européen Georges Pompidou, Service de Pneumologie, Paris, France. ${ }^{9}$ Université de Paris, Assistance Publique-Hôpitaux de Paris, Hôpital BichatClaude Bernard, Service de Pneumologie, Paris, France. ${ }^{10}$ Université Paris 13, INSERM UMR 1272 "Hypoxie et Poumon", Assistance Publique-Hôpitaux de Paris, Hôpital Avicenne, Service de Pneumologie, Bobigny, France.

${ }^{11}$ Sorbonne Université, Assistance Publique-Hôpitaux de Paris, Hôpital Tenon, Service de pneumologie et Centre Constitutif des Maladies Pulmonaires Rares, Paris, France.

Correspondence: Constance de Margerie-Mellon, Service de Radiologie, Hôpital Saint-Louis, 1, avenue Claude Vellefaux, 75010 Paris, France. E-mail: constancedemm@gmail.com

Received: 02 Jan 2019 | Accepted after revision: 04 Oct 2019

Conflict of interest: C. de Margerie-Mellon reports grants from Institut Servier and Olea Medical, outside the submitted work. L. Belin has nothing to disclose. S. Boussouar has nothing to disclose. P. Khafagy has nothing to disclose. M-P. Debray reports personal fees and non-financial support for lectures and meeting attendance from Boehringer Ingelheim and Roche, outside the submitted work. K. Levand has nothing to disclose. M-L. Chabi has nothing to disclose. A. Khalil has nothing to disclose. A. Benattia has nothing to disclose. D. Israël-Biet has nothing to disclose. B. Crestani reports personal fees and non-financial support from AstraZeneca, grants, personal fees and non-financial support from Boehringer Ingelheim and grants from MedImmune, personal fees from Sanofi, outside the submitted work. H. Nunes reports grants and personal fees for consultancy and research from Roche/Genentech, grants and personal fees for research from Boehringer Ingelheim, outside the submitted work. J. Cadranel has nothing to disclose. P. Grenier has nothing to disclose. D. Valeyre reports personal fees for advisory board work and meeting attendance from Boehringer Ingelheim and Roche, outside the submitted work. J-M. Naccache has nothing to disclose. P-Y. Brillet has received personal fees for teaching actions from Boehringer Ingelheim and Roche.

Support statement: This work was supported by the Chancellerie des Universités de Paris (Legs Poix). Funding information for this article has been deposited with the Crossref Funder Registry.

\section{References}

1 Lynch DA, Sverzellati N, Travis WD, et al. Diagnostic criteria for idiopathic pulmonary fibrosis: a Fleischner Society white paper. Lancet Respir Med 2018; 6: 138-153.

2 Raghu G, Remy-Jardin M, Myers JL, et al. Diagnosis of idiopathic pulmonary fibrosis. An official ATS/ERS/JRS/ ALAT clinical practice guideline. Am J Respir Crit Care Med 2018; 198: e44-e68.

3 Hansell DM, Bankier AA, MacMahon H, et al. Fleischner Society: glossary of terms for thoracic imaging. Radiology 2008; 246: 697-722.

4 Conger AJ. Integration and generalization of kappas for multiple raters. Psychol Bull 1980; 88: 322-328. 
5 Beigelman-Aubry C, Hill C, Guibal A, et al. Multi-detector row CT and postprocessing techniques in the assessment of diffuse lung disease. Radiographics 2005; 5: 1639-1652.

6 Johkoh T, Sumikawa H, Fukuoka J, et al. Do you really know precise radiologic-pathologic correlation of usual interstitial pneumonia? Eur J Radiol 2014; 83: 20-26.

7 Staats $\mathrm{P}$, Kligerman S, Todd $\mathrm{N}$, et al. A comparative study of honeycombing on high resolution computed tomography with histologic lung remodeling in explants with usual interstitial pneumonia. Pathol Res Pract 2015; 211: 55-61.

8 Seibold MA, Smith RW, Urbanek C, et al. The idiopathic pulmonary fibrosis honeycomb cyst contains a mucocilary pseudostratified epithelium. PLoS One 2013; 8: e58658.

9 Piciucchi S, Tomassetti S, Ravaglia C, et al. From "traction bronchiectasis" to honeycombing in idiopathic pulmonary fibrosis: a spectrum of bronchiolar remodeling also in radiology? BMC Pulm Med 2016; 16: 87.

Copyright @eERS 2020 